\title{
NOTAS SOBRE ENTEROSORA BAKER (FILICALES: GRAMMITIDACEAE) EN COSTA RICA
}

\author{
Alexander Fco. Rojas Alvarado \\ Jardín Botánico Lankester, Universidad de Costa Rica, apdo. 1031-7050, Cartago, Costa Rica. \\ afrojasa@yahoo.com
}

\begin{abstract}
Two entities of Enterosora Baker (Grammitidaceae) previously supposed to be hybrids are considered here as valid species: the new species E. bishopii A. Rojas, and the new combination $E$. enterosoroides (H. Christ) A. Rojas, based on their fertile condition, different geographical and altitudinal distribution, and distinctive morphology.
\end{abstract}

Resumen. Dos taxa de Enterosora Baker (Grammitidaceae), considerados hasta ahora como híbridos, se tratan aquí como especies válidas: E. bishopii A. Rojas, sp. nova, y E. enterosoroides (H. Christ) A. Rojas, comb. nova, con base en la condición fértil, distribución geográfica y altitudinal diferentes y morfología distinta.

Palabras clave / Kew words: Pteridophyta, Grammitidaceae, Enterosora bishopii, Enterosora enterosoroides, Costa Rica.

El género Enterosora fue establecido por Baker (1886) basado en el tipo de E. cambellii Baker. Sin embargo, por mucho tiempo algunos autores, como Lellinger (1989), han usado para este género el nombre Glyphotaenium J.Sm., el cual está basado en una ilustración que definitivamente no corresponde a Enterosora (Bishop \& Smith 1992). Bishop \& Smith (1992), en la revisión del género, combinan varias especies con el nombre Enterosora; en este mismo trabajo los autores indican la existencia de híbridos putativos entre E. cambellii Baker subsp. spongiosa (Maxon) L.E. Bishop y E. trifurcata (L.) L.E. Bishop. Ellos citan especímenes de Costa Rica: P. Standley \& J. Valerio 51633 (GH, US), R. Evans \& Bowers 2646 (MO), W. Burger \& T. Antonio 11035 (F), L. Gómez 2234-A (F), M. Grayum 7339 (MO), P. Standley \& J. Valerio 51622 (US), R. Stolze 1609 (F) y A. Brade \& C. Brade 737 (B), así como de Panamá: K. Sytsma et al. 4962 (MO), E. Killip 5284 (US), Luer et al. 10548 (MO) y G. McPherson 13592A (MO). Otro híbrido considerado fue E. percrassa (Baker) L.E. Bishop x E. trifurcata, también mencionando especímenes de Costa Rica: J. Mickel 2931 (US), R. Lent 573 (F), M. Grayum 7956(MO), A. Brade 107 (S), L. Gómez 20279 (MO, UC), A. Jiménez 2275 (F), J. Valerio 135 (US), E. Bishop 831 (UC), A. Bishop 820 (UC), A. Brenes 3726 (F), C. Horich s.n. (UC), D. Lellinger 1395 (US), P. Standley 33073 (US), J. Utley \& K. Utley 2963 (F) y C. Wercklé 660 (US), y de Panamá: A. Smith et al. 2401 (UC), H. Werff \& C. Hardeveld 6674 (MO, UC) y J. Folsom \& J. Edwards 3400 (MO). Smith \& Bishop (1995) de nuevo insisten en la existencia de los híbridos mencionados, pero sin darles nombres formales.
Los estudios sobre la pteridoflora costarricense que el autor se encuentra desarrollando, apoyado en la revisión de los ejemplares de los supuestos híbridos depositados en algunos de los herbarios nacionales [Herbario Nacional de Costa Rica (CR) y del Instituto Nacional de Biodiversidad (INB)] y con la ayuda de distintos tratamientos taxonómicos genéricos o regionales (Lellinger 1989, Bishop \& Smith 1992, Smith 1990, Smith \& Bishop 1995), sirvieron para llegar a la conclusión de que las entidades mencionadas son especies válidas, separables por su morfología, condición fértil y diferente distribución geográfica y altitudinal.

\section{Enterosora bishopii A. Rojas, sp. nova \\ Enterosora symmicta L.E. Bishop, nom. herbar.}

A Enterosora trifurcata (L.) L.E. Bishop similis, sed stipite longiore, lamina lanceolata, segmentis obtusis et soris costalibus elongatis recedit.

TIPO: COSTA RICA. Limón: Talamanca; Bratsi, Amubri, Alto Lari, Kibut, siguiendo la fila divisoria entre Ríos Lari y Dapari, cuenca superior de ambos, $9^{\circ} 22^{\prime} 45^{\prime} \mathrm{N}, 8^{\circ} 06^{\prime} 15^{\prime \prime} \mathrm{W}, 1900 \mathrm{~m}, 24$ mar 1992, G. Herrera 5478 (Holotipo: CR). Fig. 1.

Epífita; rizoma 2-5 mm de diámetro, compacto; escamas del rizoma de $3-5 \times 0.3-0.8 \mathrm{~mm}$, lanceoladas, doradas, lustrosas, por lo general irregularmente dentadas; frondas de 9-37 cm de largo, medianamente péndulas; estípite de $5-18 \mathrm{~cm} \times 0.5-1.2 \mathrm{~mm}, 1 / 3-$ 2/5 del tamaño de la fronda, atropurpúreo a negro, 
lustroso, setoso a glabrescente, pero comúnmente con muchas setas en la base del estípite, éstas de 1$2 \mathrm{~mm}$ de largo, atropurpúreas; lámina de 6-18 x 2$5.5 \mathrm{~cm}, 3.5-5$ veces más larga que ancha, lanceolada a lanceolado-oblonga, esponjosa, base obtusa a cuneada, ápice obtuso a agudo, incisa 1/4-3/5 de la longitud entre el margen y la costilla media, con lóbulos redondeados a obtusos, ascendentes $60^{\circ}-80^{\circ}$ de la costilla media, sorífera a todo lo largo o con la base y el ápice estériles, dispersamente setosa o glabra a lo largo de los márgenes y de la costilla media, las setas de 0.5-1.5 mm de largo, esparcidas en los soros; nervaduras ocultas, principalmente areoladas a lo largo de la costilla media, libres o raramente areoladas hacia los márgenes; soros en su mayoría 2-6 pares por lóbulo, redondeados, alargados cerca de la costilla media, leve a profundamente hundidos en fóveas redondeadas a alargadas, con bordes inclinados o casi rectos; esporangios glabros.

Distribución. Desde (1200-) 1540 hasta 2100 (-2500) m. Hasta ahora sólo conocida de la Cordillera Central y Cordillera de Talamanca en Costa Rica y Panamá.

Paratipos. COSTA RICA. Las Palmas, 2500 m, jun 1912, O. Jiménez 660 (CR). Guanacaste: Parque Nacional Rincón de La Vieja, Liberia, cabeceras de Quebrada Provisión y Rancho Grande, Meseta Aguacatales, $10^{\circ} 46^{\prime} \mathrm{N}, 85^{\circ} 49^{\prime} \mathrm{W}, 1350-1400 \mathrm{~m}, 1$ dic 1987, G. Herrera 1457 (CR). Heredia: Río Vueltas (upper Río Patria), E slope of Volcán Barva, near the continental divide, $10^{\circ} 06^{\prime} \mathrm{N}, 84^{\circ} 04^{\prime} \mathrm{W}$, about $2000 \mathrm{~m}$, 2 Jul 1983, K. Barringer \& E. Christenson 3376 (CR); Volcán Barva, 2000 m, 10 May 1969, A. Goldgewicht \& L. Gómez s.n. (CR); Río Las Vueltas, 2100 m, 10 mayo 1969, L. Gómez 820 (CR), L. Gómez \& A. Goldgewicht 820A (CR), L. Gómez \& A. Goldgewicht $820 B$ (CR), L. Gómez \& A. Goldgewicht 821 A (CR), L. Gómez \& A. Goldgewicht $821 B$ (CR); Braulio Carrillo National Park, $10^{\circ} 15^{\prime} \mathrm{N}, 84^{\circ} 10^{\prime} \mathrm{W}, 1830 \mathrm{~m}, 1$ Febr 1988, E. Hennipman et al. 6785 (CR), muestra mixta con E. campbelli var. spongiosa; bordes del Río Grande, afluente del Río Patria, en el paso del Gallito, macizo del Volcán Barva, 2045 m, 14 ago 1964, A. Jiménez 2275 (CR). Limón: Talamanca, Cordillera de Talamanca, Parque Internacional La Amistad, entre Quebrada Kuisa y Río Lori, camino de Ujarrás a San José Cabécar, 9²0’25'N, 8313'45”W, 2100 m, 17 mar 1993, G. Herrera 5910 (CR); Limón, El Progreso, Fila Matama, Valle de La
Estrella, 947'20”N, 8307'30”W, 1600 m, 24 abr 1989, G. Herrera \& A. Chacón 2770 (CR); Siquirres, Las Brisas de Pacuarito, subiendo por la fila superior de la margen izquierda de la Quebrada Jesús hasta

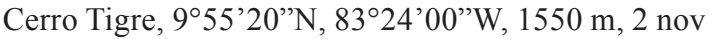
1995, G. Herrera et al. 8682 (CR, MO). Puntarenas: Reserva Biológica Monteverde, Ojo de Agua, $10^{\circ} 15^{\prime} \mathrm{N}, 84^{\circ} 46^{\prime} \mathrm{W}, 1600$ m, 20 abr 1989, E. Bello 793 (CR); Reserva Biológica Monteverde, Pantanoso Trail, 10 May 1988, S. Bigelow \& P. Kukle 116 (CR, MO); Monteverde, Sendero Pantanoso, 1540 m, 25 Nov 1979, S. Koptur 240 (CR).

PANAMÁ. Bocas del Toro: NW ridge of Cerro Pate Macho from summit to Finca Serrano, 1200-2100 m, 27 May 1981, K. Sytsma et al. 4962 (CR).

Enterosora bishopii difiere de E. trifurcata (L.) L.E. Bishop por tener un estípite de $1 / 3-2 / 5$ de la longitud de la fronda [(vs. 1/6-1/4 (-1/3)], lámina lanceolada (vs. linear a linear-oblonga), 3.5-5 veces más larga que ancha [vs. (5-) 7-12 veces más larga que ancha] e incisa 1/4-3/5 entre el margen y la vena [vs. (3/5-) 2/3-3/4], segmentos obtusos (vs. agudos), soros con setas interesporangiales (vs. sin setas) y los soros costales elongados (vs. redondos). Difiere de E. campbellii Baker subsp. spongiosa (Maxon) L.E. Bishop por tener un estípite atropurpúreo a negro (vs. pardo claro a pardo), lámina incisa 1/4$3 / 5$ entre el margen y la vena (vs. 1/3-4/5) y soros costales ovados a oblongos y el resto redondos (vs. todos lineales) (Fig. 1).

Los especímenes de herbario muestran que Enterosora campbellii subsp. spongiosa es extremadamente escasa y distribuida entre 1400 y $2500 \mathrm{~m}$ y no precisamente ubicada en lugares donde existen $E$. trifurcata [distribuida entre 300 y $1400(-1700) \mathrm{m}$ ] y la especie nueva, como para que se justifique que la especie aquí descrita es un híbrido entre las especies mencionadas. Aún si la morfología muestra caracteres intermedios, esto podría indicar que la especie es de origen híbrido, probablemente alopoliploides estabilizados como lo indican Smith y Bishop (1995), pero esta especie es fértil y más abundante que $E$. cambellii, lo cual resulta contradictorio, ya que los híbridos suelen ser estériles o más escasos que sus progenitores. 
Etimología. Esta especie es dedicada a Earl Bishop por sus múltiples contribuciones al estudio del género Enterosora.

\section{Enterosora enterosoroides (H. Christ) A. Rojas, comb. nova}

Polypodium enterosoroides H. Christ, Bull. Herb. Boissier, ser. 2, 7(4): 260. 1907.

Tipo: Costa Rica, La Palma, 1500 m, C. Wercklé 17095 [Holotipo: P (n.v.), isotipos: CR!, US!)].

Epífitas; rizoma claramente robusto, las escamas hasta 4 x $0.8 \mathrm{~mm}$, lanceoladas, pardo-doradas a parduscas, lustrosas, en su mayoría sinuado-dentadas, eciliadas o con tricomas glandulares marginales esparcidos; estípite 2-8 cm x 0.3-0.6 mm, 1/4-2/5 de la longitud de la fronda, pardo oscuro a negro, lustroso, variablemente setoso o glabrescente, generalmente flexionado en el ápice; lámina de 8-15 x 1-2 cm, 58 veces más larga que ancha, entera a crenadamente incisa hasta $1 / 3(-1 / 2)$ de la longitud entre el margen y la costilla media, con lóbulos ampliamente redondeados y ligeramente ascendentes, linear o linear-elíptica, engrosada y esponjosa, redondeada a aguda en ambos extremos, sin soros hacia la base, sorífera hacia el ápice o no, setosa a lo largo de los márgenes, la costilla media y alrededor de los soros; nervaduras ocultas, principalmente areoladas a lo largo de la costilla media, variablemente así hacia los márgenes; soros en su mayoría 2-4(-5) pares por lóbulo, redondeados a ovales, no extendiéndose hacia los márgenes o el ápice de los lobos, hundidos en fóveas redondeadas o más o menos alargadas con bordes inclinados.

Distribución. Cordillera de Tilarán, Cordillera Central y Cordillera de Talamanca en Costa Rica y Panamá, 1150-2000 m.

Material revisado. COSTA RICA. El Gallito, 1800 m, 20 dic 1927, M. Valerio 135a (CR). Alajuela: La Palma de San Ramón, 23-26 oct 1922, A. Brenes 7050 (CR). Cartago: Cañón del Río Grande de Orosi y aluvión, 23 oct 1983, I. Chacón et al. 1487 (CR); Turrialba, Tayutic, Jicotea, entre Río Jicotea y Río

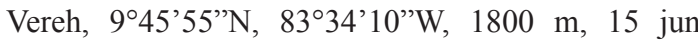
1995, G. Herrera \& I. Martínez 7904 (CR); Paraíso, Orosi, Parque Nacional Tapantí, Sendero Oropéndola, 9॰44'53”N, 8346'55'W, 1200 m, 17 jun 1993, R. Rivero 2225 (CR); Paraíso, Parque Nacional Tapantí, Cuenca del Reventazón, Estación Tapantí, Sendero
Árboles Caídos, 944'53”N, 8346'55'W, 1600 m, 10 ene 1997, A. Rodríguez et al. 1860 (CR, INB); Paraíso, Parque Nacional Tapantí, Cuenca del Reventazón, Sendero Árboles Caídos, 945'45'N, 8347'17'W, 1150-1300 m, 24 febr 1997, A. Rojas 3379 (CR, INB); Paraíso, Cuenca del Reventazón, Estación de Biología Tropical Río Macho y alrededores, 946’N, 8352’W, 1580-1800 m, 3 ago 1994, A. Rojas \& L. Mejía 1359 (CR, INB); Paraíso, Valle del Reventazón, Mirador Ecológico Monte Sky, 9`44'40”N, 8310’00”W, 17002000 m, 23 sept 1995, A. Rojas et al. 2481 (CR, INB); Paraíso, Cuenca del Reventazón, Estación de Biología Tropical Río Macho y alrededores, 9॰46’00”N , 83 $51^{\prime} 00$ 'W, 1630-1730 m, 27 sept 1996, A. Rojas et al. 3123 (CR, INB).

Enterosora enterosoroides difiere de E. trifurcata por tener un estípite de $1 / 4-2 / 5$ de la longitud de la fronda [vs. (1/3-) 1/4-1/6], lámina 5-8 veces más larga que ancha [vs. (5-) 7-12 veces más larga que ancha] y casi entera a 1/3 (-1/2) incisa [vs. incisa (3/5-) 2/3-3/4]. Difiere de Enterosora ecostata (Sodiro) L.E. Bishop por tener un estípite de 1/4-2/5 de la longitud de la fronda [vs. (1/4-) 1/5-1/8], atropurpúreo a negro (vs. pardo claro a pardo) y lámina 5-8 veces más larga que ancha [vs. 7-15 veces más larga que ancha] (Fig. 2). Si esta especie es de origen híbrido, es más probable que sus ancestros sean Enterosora ecostata y E. trifurcata, pero evidentemente se trata de una especie, ya que es fértil, existe en sitios donde no se han localizado los supuestos progenitores e incluso es más abundante que E. ecostata.

Smith \& Bishop (1995) mencionan los especímenes A. Skutch 2760 (US), a 915 m, y A. Smith et al. 2394 (MO, UC), a 1600 m, como híbridos entre Enterosora ecostata y E. percrassa (Baker) L.E. Bishop. La suposición anterior parece poco probable, ya que la primera especie existe a $100-1000 \mathrm{~m}$ y la segunda a 1800-2400 m, según lo indican los mismos autores (Smith \& Bishop 1995). Estos especímenes referidos no han sido vistos, pero por la distribución altitudinal y por la morfología descrita no parecen ser diferentes a E. enterosoroides. En observaciones de campo, en el Parque Nacional Tapantí, se ha notado que las plantas juveniles o poco desarrolladas pueden tener la lámina entera o apenas levemente lobada, más lineal, más densamente setosa y las setas más largas, asemejándose a E. ecostata, la cual no se encuentra dentro del ámbito geográfico ni altitudinal de E. enterosoroides. 
Agradecimientos. Agradezco a los herbarios del Museo Nacional de Costa Rica (CR) e Instituto Nacional de Biodiversidad (INB) por permitirme utilizar sus colecciones, a Franco Pupulin por la diagnosis latina, a Carlos O. Morales por sus correcciones y a los revisores anónimos por sus comentarios, que mejoraron la calidad de este escrito.

\section{LITERATURA CITADA}

Baker, J.G. 1886. Enterosora. In: Im Thurn, E.F. (ed.). Notes on the plants observed during the Roraima Expedition of 1884. Timehri 5: 218.
Bishop, L.E. \& A.R. Smith. 1992. Revision of the fern genus Enterosora (Grammitidaceae) in the New World. Syst. Bot. 17(3): 345-362.

Lellinger, D.B. 1989. The ferns and fern-allies from Costa Rica, Panama, and The Chocó. Part I. Pteridologia. 364 p.

Smith, A.R. 1990. Pteridophytes of the Venezuelan Guayana: new species. Ann. Missouri Bot. Gard. 77: 249-273.

Smith, A.R. \& L.E. Bishop. 1995. Enterosora. In: Moran, R.C. \& R. Riba (eds.). Flora Mesoamericana. Vol. 1. Psilotaceae a Salviniaceae. Univ. Nac. Autón. México. México, D.F. p. 372-375.

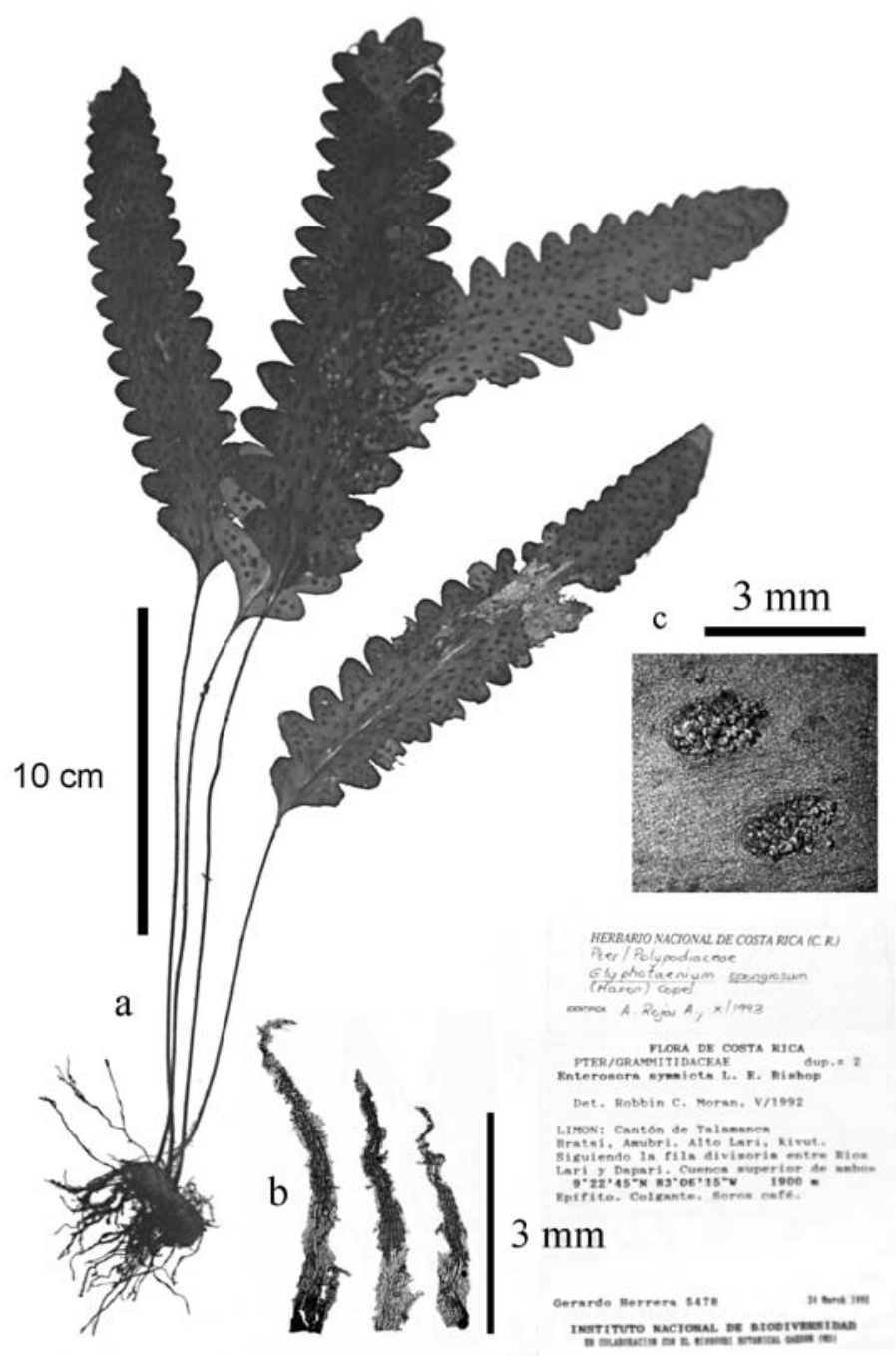

Fig. 1. Ejemplar tipo de Enterosora bishopii A. Rojas. (G. Herrera 5478, CR). a) Hábito. b) Escamas del rizoma. c) Detalle de la lámina. 


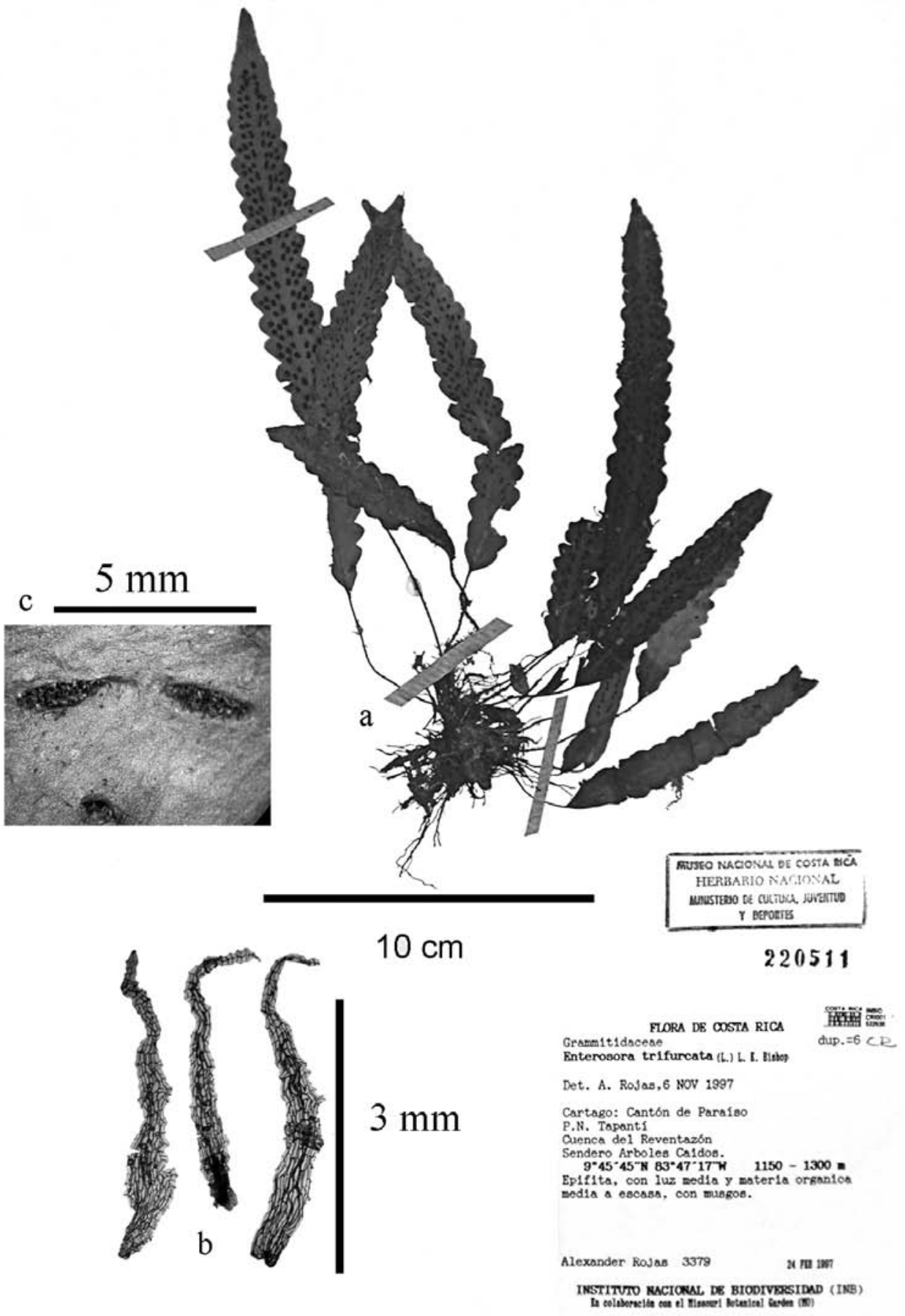

Fig. 2. Ejemplar representativo de Enterosora enterosoroides (H. Christ) A. Rojas. (A. Rojas 3379, CR). a) Hábito. b) Escamas del rizoma. c) Detalle de la lámina. 
